CrossMark \& click for updates

Cite this: Phys. Chem. Chem. Phys., $2017,19,8496$

Received 16th December 2016, Accepted 27th February 2017

DOI: $10.1039 / c 6 c p 08589 j$

rsc.li/pccp

\section{In situ probing of the crystallization kinetics of rr-P3HT on single layer graphene as a function of temperature $\dagger$}

\author{
Nicolas Boulanger, ${ }^{a}$ Victor $Y u,{ }^{b}$ Michael Hilke, ${ }^{b}$ Michael F. Toney ${ }^{c}$ and \\ David R. Barbero*a
}

\begin{abstract}
We studied the molecular packing and crystallization of a highly regio-regular semiconducting polymer poly(3-hexylthiophene) (P3HT) on both single layer graphene and silicon as a function of temperature, during cooling from the melt. The onset of crystallization, crystallites' size, orientation, and kinetics of formation were measured in situ by synchrotron grazing incidence X-ray diffraction (GIXD) during cooling and revealed a very different crystallization process on each surface. A favored crystalline orientation with out of plane $\pi-\pi$ stacking formed at a temperature of $200{ }^{\circ} \mathrm{C}$ on graphene, whereas the first crystallites formed with an edge-on orientation at $185^{\circ} \mathrm{C}$ on silicon. The crystallization of face-on lamellae revealed two surprising effects during cooling: (a) a constant low value of the $\pi-\pi$ spacing below $60{ }^{\circ} \mathrm{C}$; and (b) a reduction by half in the coherence length of face-on lamellae from 100 to $30{ }^{\circ} \mathrm{C}$, which corresponded with the weakening of the 2 nd or $3 r d$ order of the in-plane $(k 00)$ diffraction peak. The final ratio of face-on to edge-on orientations was $40 \%$ on graphene, and $2 \%$ on silicon, revealing the very different crystallization mechanisms. These results provide a better understanding of how surfaces with different chemistries and intermolecular interactions with the polythiophene polymer chains lead to different crystallization processes and crystallites orientations for specific electronic applications.
\end{abstract}

\section{Introduction}

Flexibility, ultrafast charge transport, and the possibility to produce mono-atomically thin layers of graphene on large areas by chemical vapor deposition (CVD), ${ }^{1}$ has accelerated the use of graphene in electronic devices such as photovoltaic devices, light emitting diodes, and nonvolatile memory devices..$^{2-4}$ Another advantageous property of graphene is that it can be combined with a thin layer of an organic semiconductor to produce organic hybrid optoelectronic devices. ${ }^{5,6}$ Efficient transfer of charges at the interface between graphene and a thin layer of conjugated organic semiconductor, has been shown to occur through $\pi-\pi$ interactions. ${ }^{4,7,8}$ It was moreover recently demonstrated that combining graphene and a semiconducting polymer such as poly-3-hexylthiophene (P3HT) produced enhanced charge transport in the vertical direction, and could help the development of faster and more efficient opto-electronic devices such as OPVs and OLEDs. ${ }^{9}$

\footnotetext{
${ }^{a}$ Nano-Engineered Materials and Organic Electronics Laboratory, Umeå Universitet, Umeå 90187, Sweden. E-mail: david.barbero@umu.se

${ }^{b}$ Department of Physics, McGill University, Montréal, Québec, H3A 2T8, Canada

${ }^{c}$ Stanford Synchrotron Radiation Lightsource, Menlo Park, CA, USA

$\dagger$ Electronic supplementary information (ESI) available: Details about the preparation of the single layer graphene as well as the grazing incidence X-ray diffraction measurements. See DOI: 10.1039/c6cp08589j
}

However, the crystallinity of the polymer film strongly influences charge transport in the active layer of an electronic device, which will finally impact its overall efficiency. Therefore, in order to predict and control the electronic properties of a single sheet graphene/semiconducting polymer film layer, it is important to understand how the polymer crystallizes on a mono-layer of graphene, and how different crystallization on graphene is from a typical substrate, e.g. silicon or silicon oxide. Indeed, the crystallization of a semiconducting polymer, such as P3HT, depends on several factors: interaction with the substrate, annealing temperature, rate of cooling, etc. Although P3HT has been widely studied on more common (and often weakly interacting) surfaces, e.g. silicon oxide, there is still very little published regarding the crystallization of $\mathrm{P} 3 \mathrm{HT}$ and other semiconducting polymers on single layer graphene. ${ }^{10-13}$ It has previously been shown that the crystalline order of P3HT can be affected by changing the chemistry of the substrate, as was previously shown by spin coating the polymer on substrates treated with either hexamethyldisilizane or octadecyltrichlorosilane. ${ }^{14}$

Here, we show a detailed in situ study of the kinetics and process of crystallization of a $85 \mathrm{~nm}$ thick P3HT film spun from orthodichlorobenzene $(o-\mathrm{DCB})$ on both graphene and silicon (with native oxide) as a function of temperature during slow cooling from the melt. We discuss our results in the light of a previous study of thin 
P3HT films (20-25 nm) spun from chloroform onto silicon, where the (200) layer spacing and peak width were measured in situ during heating and cooling. ${ }^{16}$ Our findings reveal several differences with this previous study, but also clearly show that the way crystallites form on graphene is fundamentally different than on the less interacting silicon surface, which results in different crystallite orientations, kinetics of crystallization, and a very different final ratio of face-on to edge-on lamellae, which imply largely different opto-electronic properties. The influence of the surface chemistry on the crystalline structure of polymer films was previously observed. ${ }^{14}$

\section{Experimental}

\section{Materials}

Poly-3-hexylthiophene (P3HT) $\left(98 \% \quad \mathrm{RR}, \quad M_{\mathrm{w}}=\begin{array}{llll}32 & \mathrm{kD}\end{array}\right)$ was purchased from American Dye Source Inc. Single layer graphene was produced by CVD on a copper foil and subsequently transferred to a solid substrate (silicon or glass). Details of the synthesis and transfer procedure of graphene, and other materials used can be found in the ESI. $\dagger$

\section{Sample preparation and characterization}

Thin films of regio-regular P3HT (98\%) (85 $\mathrm{nm} \pm 3 \mathrm{~nm}$ as measured by AFM and shown in Fig. S1 in ESI $\dagger$ ) were deposited on both graphene and silicon (with native oxide) substrates by spin-coating of a dilute solution of $\mathrm{P} 3 \mathrm{HT}$ in ortho-dichlorobenzene (o-DCB). They were then placed in an inert atmosphere (helium) chamber and heated up to $240{ }^{\circ} \mathrm{C}$ and maintained there for $5 \mathrm{~min}$, before slow cooling $\left(2-3{ }^{\circ} \mathrm{C} \min ^{-1}\right)$, where they were analyzed in situ by synchrotron X-ray diffraction at the 11-3 beamline at the Stanford Synchrotron Radiation Lightsource (SSRL), as schematized in Fig. 1A and B. This method gives accurate information about the crystalline structure, the crystallites orientation and their coherence length inside the films.

\section{Results and discussion}

Sheets of graphene synthesized by chemical vapor deposition were transferred onto the silicon substrates by a floating method as detailed elsewhere. ${ }^{9,17}$ These were recently characterized by
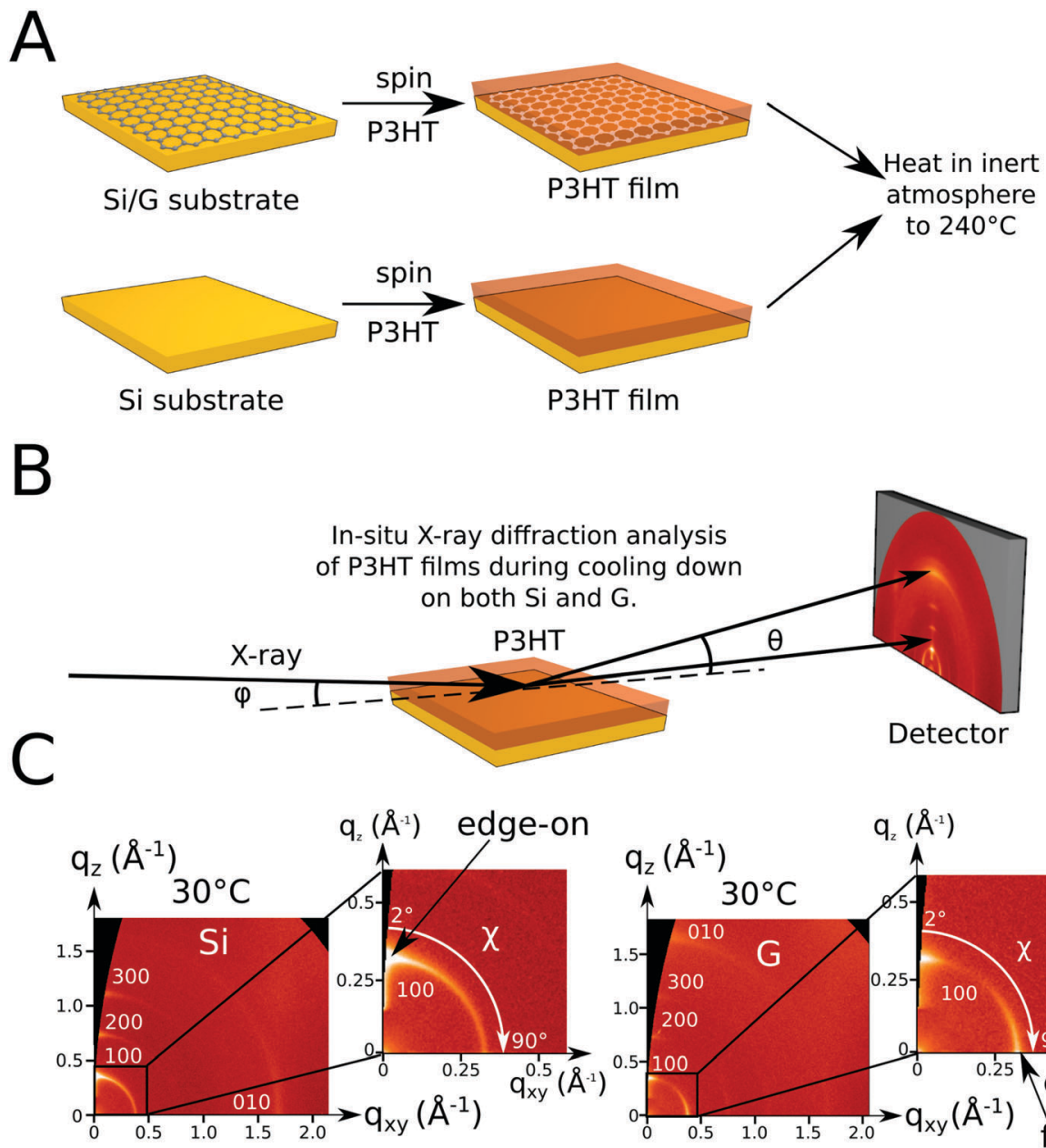

Fig. 1 Preparation of the graphene/P3HT samples. (A) Thin films of P3HT were spun from solution onto either silicon or a single layer graphene surface, heated up to $240{ }^{\circ} \mathrm{C}$ for $5 \mathrm{~min}$ in inert atmosphere, and slowly cooled down to room temperature. (B) During cooling down, the films were characterized by grazing incidence $X$-ray diffraction (GIXD), and their crystallization monitored at various temperatures using a planar detector. (C) $2 \mathrm{D}$ grazing incidence diffraction patterns of P3HT films on Si and on graphene, showing $q_{z}, q_{x y}$ and the angle $\chi$. 
Raman spectroscopy which confirmed that these sheets were monolayers of graphene. ${ }^{9}$

The thin films of P3HT which was spun on silicon and graphene substrates were heated to $240{ }^{\circ} \mathrm{C}$, above their melting point $\left(T_{\mathrm{m}} \approx 230{ }^{\circ} \mathrm{C}\right)$, in order to ensure complete vanishing of crystallites formed during spin-coating. Fig. S2 (ESI $\dagger$ ) shows grazing incidence X-ray diffraction (GIXD) snapshots of both films on silicon ( $\mathrm{Si}$ ) and graphene $(\mathrm{G})$ at different temperatures during cooling. At $240{ }^{\circ} \mathrm{C}$, no diffraction was detected in either film, indicating a disordered state. It is possible that some partial ordering is still present in the form of small aggregates in the film.

Upon cooling, crystallites started to form and became visible in the diffraction patterns. Close to room temperature, at $30{ }^{\circ} \mathrm{C}$, the crystalline structure in both films showed clear differences (Fig. 1C) with different crystalline orientations and in various amounts. Both samples contained edge-on lamellae (characterized by the strong (100) diffraction peak along $q_{z}$ ), as schematically showed in Fig. 2B, where the insulating hexyl side chains shown Fig. 1A are oriented perpendicularly to the substrate. Because it can readily form edge-on lamellae on select substrates, P3HT has been one of the most studied materials for organic based FETs where charges are transported along the in-plane $\pi-\pi$ stacking. ${ }^{18-21}$ Formation of mainly edge-on lamellae and the absence of (010) peak near the $z$ axis (vertical $\pi$ stacking) is often observed on $\mathrm{Si}$ and weakly interacting substrates. ${ }^{16,22-25}$ The solvent and time of film growth were found to be important, and usually longer crystallization times and high boiling point solvent have been reported to favor edge-on growth on silicon. This observation is consistent with our experimental conditions which were meant to simulate a thermodynamic equilibrium in the melt with very slow cooling. Calculations and simulations have also shown the lower energetic cost of forming edge-on crystallites on silicon compared to face-on for polythiophene and other polymers. ${ }^{26-28}$

Face-on lamellae, with $\pi-\pi$ stacking pointing along the [010] direction (see Fig. 2C) were also present mostly on the graphene sample (strong diffraction spots in $(100)_{x y}$ in $x y$ plane, and in $(010)_{z}$ near the $z$ axis). The vertical $\pi-\pi$ stacking has been reported to favor charge transport perpendicularly to the substrate, and is considered to be beneficial for opto-electronic diode and solar cell devices. ${ }^{29-32}$ Recently, the use of single layer graphene was shown to promote face-on orientation and to enhance out-of-plane conductivity and mobility in P3HT compared to silicon, reaching a mobility $\mu \approx 2.8 \times 10^{-4} \mathrm{~cm}^{2} \mathrm{~V}^{-1} \mathrm{~s}^{-1} .9$

Fig. 3A represents the evolution of the amount of edge-on lamellae during cooling at a $2-3{ }^{\circ} \mathrm{C} \mathrm{min}^{-1}$ rate on $\mathrm{Si}$ and $\mathrm{G}$, and showed that these grew much faster, and reached a much larger amount, on the silicon substrate. By comparison, the final amount of edge-on lamellae on graphene were $\approx 19 \%$ of that on silicon at $30{ }^{\circ} \mathrm{C}$. We defined the face-on ratio $(F)$ as the intensity of the $(100)_{x y}$ peak divided by the sum of the intensities of both the $(100)_{x y}$ and $(100)_{z}$ peaks. The evolution of $F$ as a function of temperature is plotted in Fig. 3B. Peak intensities were extracted from fitting peak areas of line cuts from the $2 \mathrm{D}$ diffraction patterns. This panel in particular shows that the film on graphene started crystallizing with face-on lamellae only (characterized by $100 \%$ face-on ratio), before the first edge-on crystallites started to appear around $185{ }^{\circ} \mathrm{C}$. This indicates that the P3HT chains orientate themselves cofacially with the substrate due to the $\pi-\pi$ interactions with the graphene layer, instead of the preferred edge-on orientation encountered on silicon. This result is consistent with simulations of crystallization of P3HT on graphene, which showed that the strong $\pi-\pi$ interaction between the thiophene backbone and the graphene layer results in higher binding energy for the face-on orientation compared to edge-on. ${ }^{10}$ In the case of the silicon substrate, hydrophobic interactions between the substrate and the hexyl side chains results in an edge-on configuration which minimizes the free energy for crystallization compared to face-on crystallites. ${ }^{33,34}$ The final face-on ratio was $\approx 40 \%$ on graphene, whereas it was only $\approx 2 \%$ on silicon, highlighting the very different crystallization processes taking place on both surfaces.

The pole figure showed in Fig. 3C, also clearly shows the difference in the final crystalline orientations at $30{ }^{\circ} \mathrm{C}$ for both films on silicon and graphene, and represents the (100) normalized diffraction intensity (area) (see Fig. 1C). It is clearly
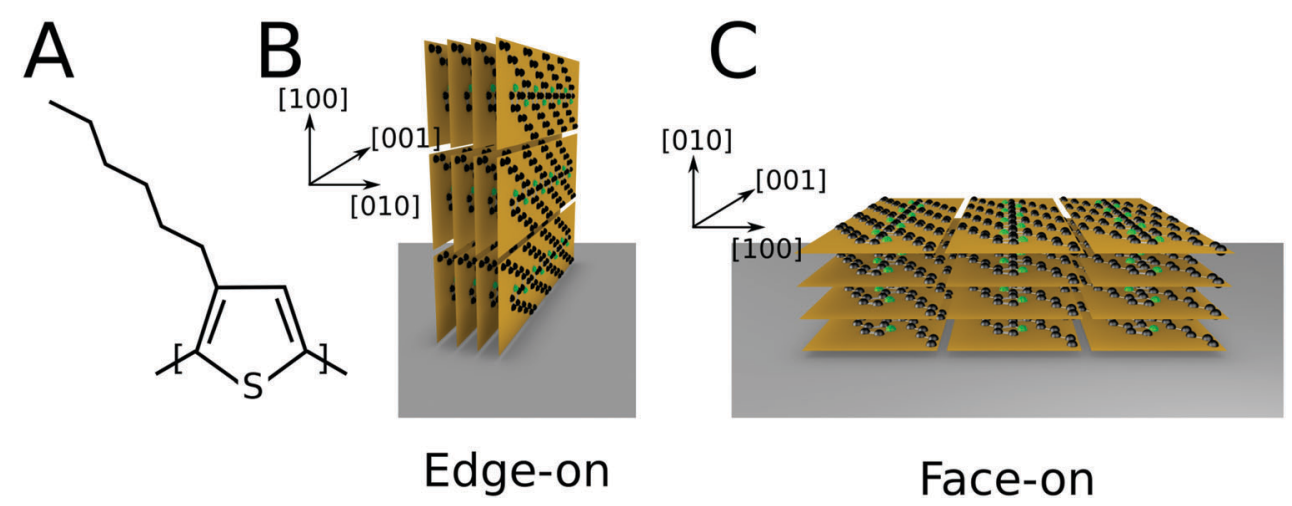

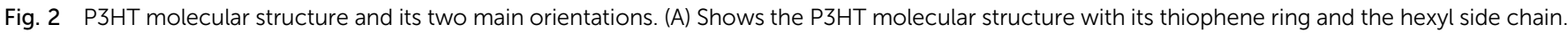

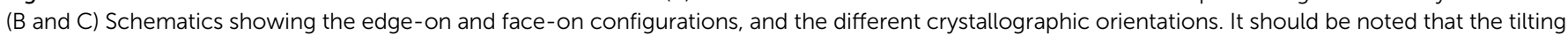
of the chains within the crystals is unknown and not represented here. ${ }^{15}$ 

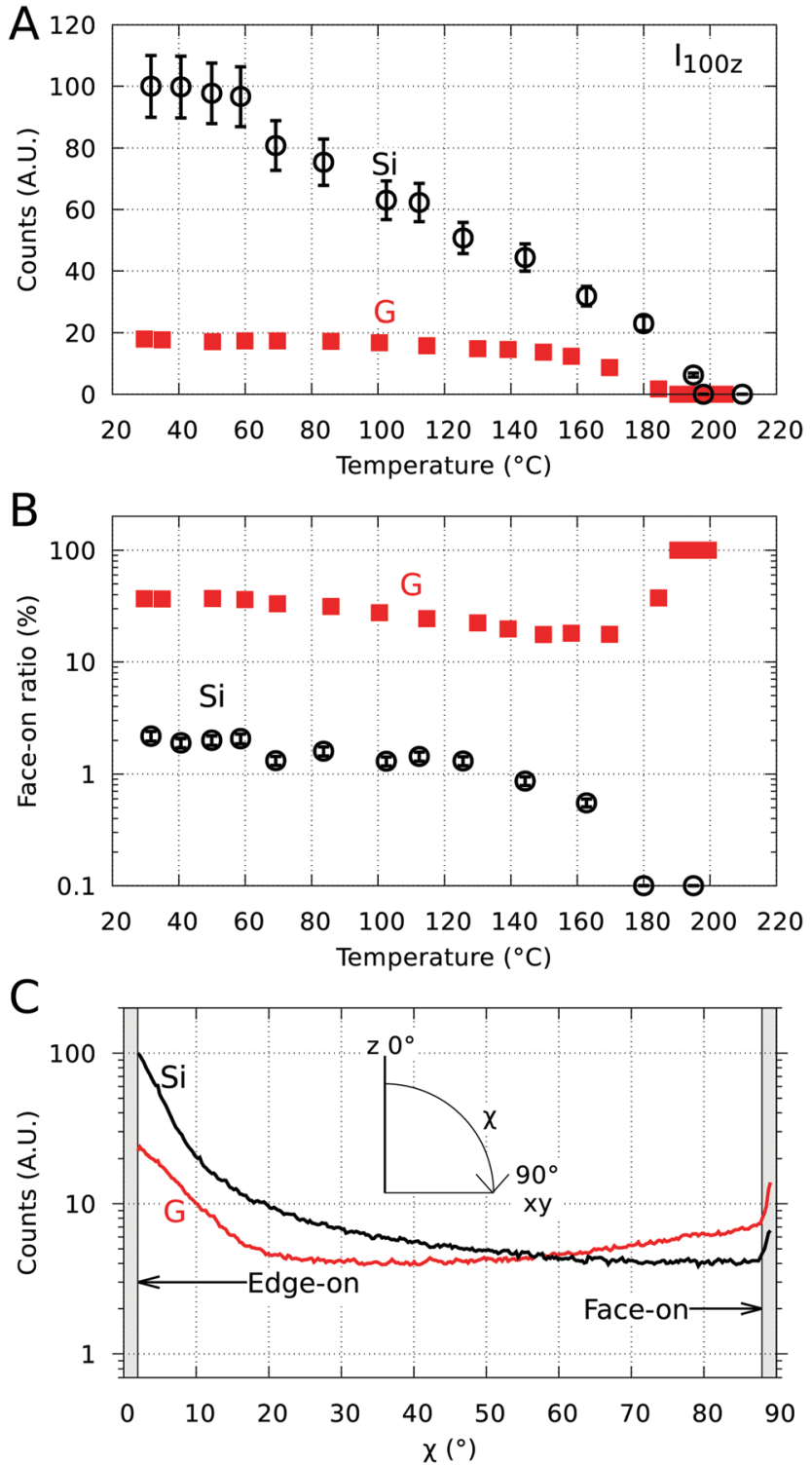

Fig. 3 (A) Intensity of the 100 peak in the out-of-plane direction as a function of temperature during cooling. (B) Log scale of the face-on ratio $(F)$ showing the diffraction intensity ratio of the signal from face-on crystallites compared to both face-on and edge-on. It is clear that the graphene sample starts crystallizing in a face-on configuration, while the film on silicon shows mostly edge-on crystallites. (C) Log scale of $\chi$ plots for both the films on graphene and silicon at $30{ }^{\circ} \mathrm{C}$, with $\chi=0^{\circ}$ corresponding to edge-on and $\chi=90^{\circ}$ being face-on, as shown in the inset. The intensities reported in (A) are obtained from peak areas fitted from line cuts of the $2 \mathrm{D}$ diffraction patterns. The face-on ratio in (B) is defined as $I_{100} /\left(I_{100}+I_{100}\right)$.

visible that the film on silicon is mostly edge-on, while the film on graphene has a much larger proportion of face-on crystallites. It is also clearly seen that at higher $\chi\left(60\right.$ to $\left.88^{\circ}\right)$, the amount of misoriented face-on lamellae is more pronounced on graphene compared to silicon (Fig. 3C).

A more detailed analysis of the nucleation and growth of crystallites on silicon $(\mathrm{Si})$ and graphene $(\mathrm{G})$ is presented in Fig. 4. During heating up, all diffraction peaks disappeared at
$240{ }^{\circ} \mathrm{C}$ on both substrates, indicating loss of ordered crystallites in the films. During slow cooling, crystallites started to form again between $\approx 200$ and $185{ }^{\circ} \mathrm{C}$, but with a different crystalline orientation on silicon and on graphene. The temperatures given take into account the accuracy of reading from the thermocouple $\left(0.1{ }^{\circ} \mathrm{C}\right)$ and the resolution during cooling (one measure every $5{ }^{\circ} \mathrm{C}$ for graphene and every $10{ }^{\circ} \mathrm{C}$ for silicon in the range 240 to $180{ }^{\circ} \mathrm{C}$ ). As the films were slowly cooled down, the formation of small crystallites became apparent in the form of weak diffraction peaks in (100) at $q=0.35 \AA^{-1}$ for the graphene substrate, $q=0.31 \AA^{-1}$ for the silicon substrate. Since it was difficult to precisely determine the onset of crystallization due to the weak diffraction intensity at temperatures close to the melting point, and for consistency, we arbitrarily set a normalized intensity value of $2 \%$ of the maximum intensity as the threshold for the onset of crystallization in the samples.

On graphene, the first crystallites with an edge-on orientation appeared between 185 and $180^{\circ} \mathrm{C}$, as shown by the small diffraction peak at $q \approx 0.324 \AA^{-1}$ along the $z$ axis (Fig. 4A). By contrast, the first face-on crystallites appeared at a higher temperature between 205 and $200{ }^{\circ} \mathrm{C}$, as seen by the diffracted intensity at $q \approx 0.354 \AA^{-1}$ along the $x y$ axis in Fig. $4 \mathrm{~B}$, and indicate a smaller interlayer $d$ spacing in the $x y$ plane compared to the $z$ direction perpendicular to the plane. The nucleation and subsequent growth of these lamellae during cooling is clearly visible in the plots of Fig. 4 .

On silicon, the first crystallites appeared with the (100) edgeon lamellar orientation (diffraction spot at $q \approx 0.327 \AA^{-1}$ along the $z$ axis) at a temperature below $195{ }^{\circ} \mathrm{C}$, and at $\approx 180{ }^{\circ} \mathrm{C}$ the $(100)_{z}$ peak is already very well defined (Fig. 4C), and much more intense and sharper than on graphene (Fig. 4A). During further cooling, the position of this peak shifted to $q \approx 0.360 \AA^{-1}$ on both surfaces, corresponding to a contraction of (100) interlayer spacing (see Fig. S3 of ESI $\dagger$ ). Moreover, it is interesting to note that contrary to the evolution of the $(100)_{x y}$ peak's width on graphene, the $(100)_{z}$ peak's width actually decreased considerably during cooling, indicating lamellar growth and a much higher coherence length as can be seen in Fig. 6. A detailed study of growth dynamics, interplanar spacing and coherence length on both surfaces is presented thereafter.

The shift in $q$ shown Fig. S3 in ESI, $\dagger$ indicates a reduction in interplanar spacing $d_{z}$ on both silicon and graphene of the edge-on lamellae along the $z$ axis. As illustrated in Fig. 5A, this reduction occurred for $d_{z} \approx 2.02$ to $1.75 \mathrm{~nm}$, and is consistent with a previous study of P3HT performed on silicon where a reduction in $d$ spacing from $\approx 1.83$ down to $1.66 \mathrm{~nm}$ was observed. ${ }^{16}$ A shift in $100_{x y}$ peak position to higher $q$ from 0.355 to $0.380 \AA^{-1}$ on graphene and from 0.359 to $0.380 \AA^{-1}$ on silicon was also observed during cooling, which indicates a reduction of interlayer $d_{x y}$ spacing of the face-on lamellae along the (100) direction in the $x y$ plane, from 1.78 to $1.66 \mathrm{~nm}$ on graphene, and follow a similar trend on silicon with nearly identical values. The values measured for $d_{x y}$ are consistently smaller by 0.1 to $0.2 \mathrm{~nm}$ compared to that measured for $d_{z}$ likely 

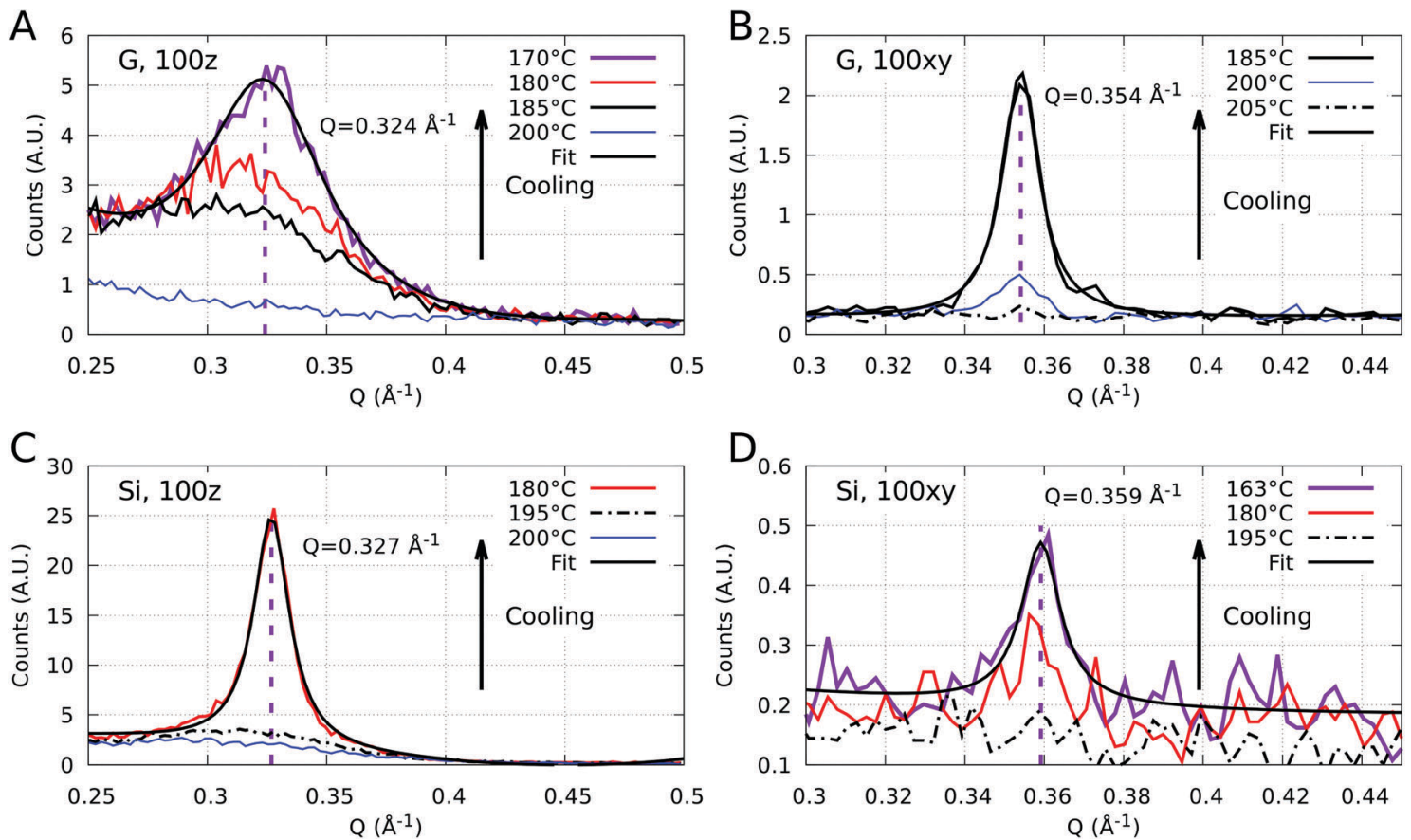

Fig. 4 In-plane $(x y)$ and out-of-plane $(z) 100$ diffraction peaks of P3HT films on graphene and on Si during cooling. The films were maintained in an inert atmosphere and probed at an incident angle of $0.13^{\circ}$. (A) Shows the evolution of the $100_{z}$ peak during cooling for P3HT on graphene, and (B) show the intensity for the $100_{x y}$ peak during cooling. The intensity of the $100_{z}$ peak, and of the $100_{x y}$ peak, during cooling on Si is shown in (C) and (D) respectively.

due to refraction effect. ${ }^{35}$ Another interesting observation concerns the reduction in $d_{z}$ of the (010) face-on lamellae on graphene. As can be seen in Fig. 5C, the (010) interlayer spacing decreases in the $z$ direction during cooling due to thermal contraction. However, surprisingly $d_{z}$ stopped decreasing and remained at a constant value of $d_{z} \approx 0.374-0.375 \mathrm{~nm}$ below $\approx 80^{\circ} \mathrm{C}$. One would expect a continuous decrease in interplanar spacing until cooling to room temperature. Similar $d$ spacing values between 3.74 and $3.81 \AA$ for the (010) face-on lamellae have been reported elsewhere in the literature on ITO, silicon, and glass. ${ }^{36-38}$ The low $d$ value measured here on graphene potentially indicates faster charge transport along the $\pi-\pi$ stacking in the vertical direction, compared to larger $\pi-\pi$ interplanar spacing.

We showed that as the polymer was cooled, the interlayer spacing decreased. It is also possible to estimate the coherence length of the crystallites from the width of the diffraction peaks as a function of temperature. The coherence length $L$ of the crystallites was then calculated from the peak FWHM $\beta$ using the Scherrer's formula:

$$
L=\frac{K \cdot \lambda}{\beta \cos \theta}
$$

where $K$ is the shape factor, estimated to $0.9, \lambda$ the X-ray wavelength $(0.974 \AA)$ and $\theta$ the Bragg angle. Fig. 6 shows the evolution of the coherence length for $(100)_{z}$ lamellae oriented in the $z$ direction, for $(100)_{x y}$ along the $x y$ plane, and for $(010)_{z}$ lamellae oriented at $\approx 8^{\circ}$ from the $z$ axis on graphene.
On silicon, edge-on crystallites grew relatively quickly, and reached $L_{100}^{Z} \approx 30 \mathrm{~nm}$ at a temperature of $180{ }^{\circ} \mathrm{C}$, just $\approx 15^{\circ} \mathrm{C}$ below the onset for crystallization of the first edge-on lamellae. The coherence length $L_{(100)}^{z}$ of the crystallites remained between $\approx 31-37 \mathrm{~nm}$ during further cooling down to $\approx 60{ }^{\circ} \mathrm{C}$, and then slightly decreased below $60{ }^{\circ} \mathrm{C}$ reaching their lowest value of $\approx 29 \mathrm{~nm}$ at room temperature. Note that no value of $L_{100}$ could be analyzed at temperatures above $180{ }^{\circ} \mathrm{C}$ due to a weak and undefined diffraction peak. On graphene, $L_{100}^{z}$ increased from zero at $180{ }^{\circ} \mathrm{C}$ to $\approx 28 \mathrm{~nm}$ at $115{ }^{\circ} \mathrm{C}$ where it remained approximately constant until $60{ }^{\circ} \mathrm{C}$ where it slowly decreased to reach $\approx 25 \mathrm{~nm}$ at room temperature. The evolution in $L_{100}^{z}$ is very similar on both silicon and graphene from 115 to $30{ }^{\circ} \mathrm{C}$, only with slightly lower values by $2-3 \mathrm{~nm}$ on graphene. Moreover, on graphene from $\approx 200{ }^{\circ} \mathrm{C}$, face-on lamellae with a coherence length $L_{010}^{z} \approx 9.5-12.5 \mathrm{~nm}$ (measured at an angle of $\approx 8^{\circ}$ from the $z$ axis) were present until $\approx 100{ }^{\circ} \mathrm{C}$. Below this temperature, the face-on coherence length quickly decreased to its final lowest value of $\approx 5.0 \mathrm{~nm}$ at $\approx 30{ }^{\circ} \mathrm{C}$, representing a $50 \%$ reduction in $L_{010}^{z}$.

The kinetics of crystallite formation are therefore very different on both surfaces, with P3HT crystallizing at higher temperatures on graphene compared to silicon. This suggests that the graphene layer acted as a seed for crystallization with a preferential face-on orientation, while a P3HT film on silicon favored an edge-on configuration. Face-on crystallites form preferably on graphene, compared to silicon, because of the $\pi$ intermolecular forces between the graphene sheet and the P3HT molecules. This molecular arrangement enables a more 


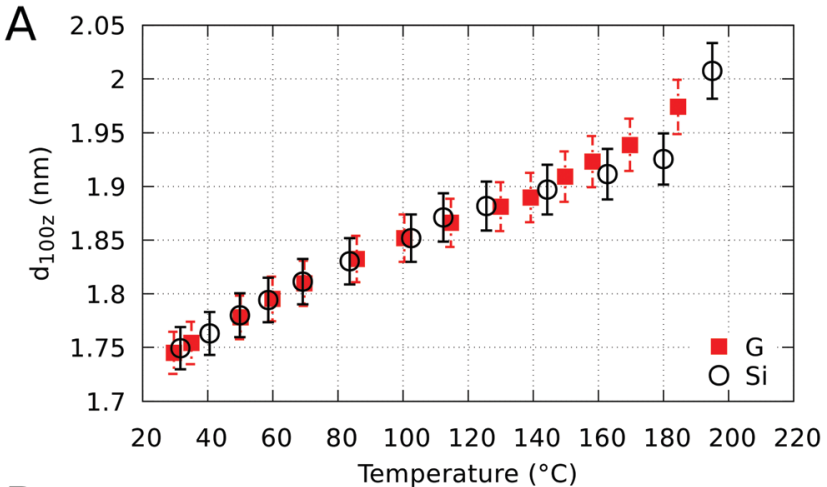

B

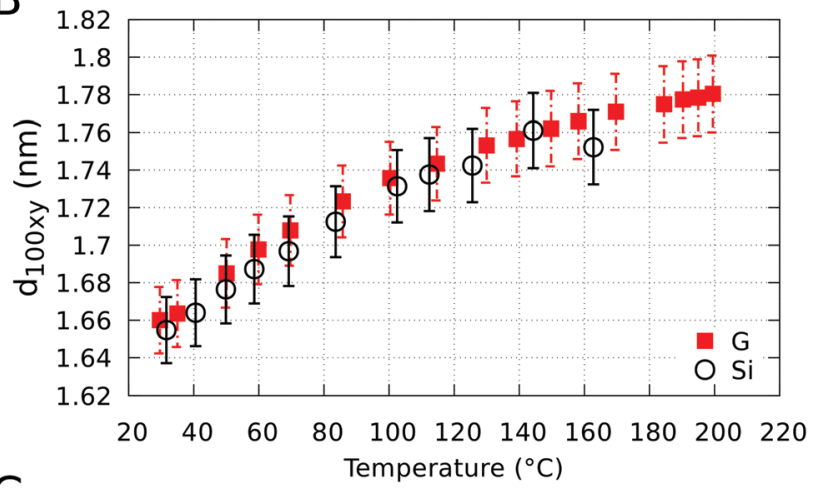

C

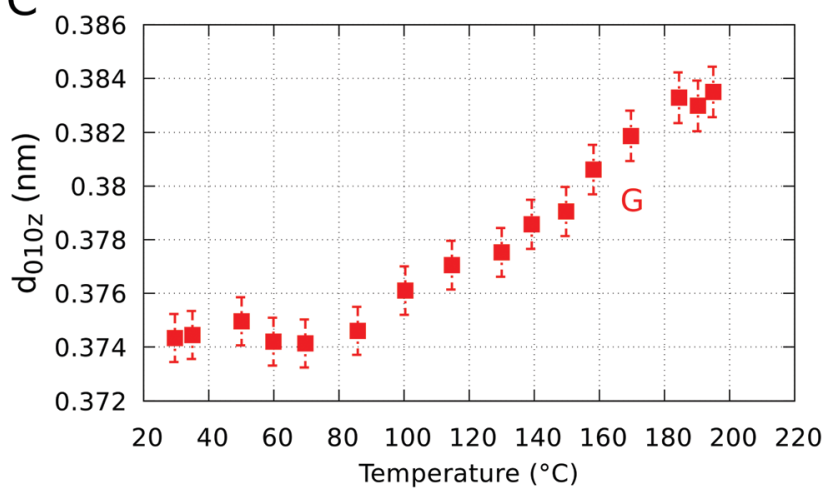

Fig. 5 Evolution of the interplanar spacing during cooling for P3HT on graphene (red squares) and Si (black circles) for (A) the out-of-plane 100 peak, (B) the in-plane 100 peak and (C) the out-of-plane 010 peak. All distances decreased with cooling, which can be attributed to thermal contraction. It should be noted that the interplanar spacing for the 010 peak reached a minimum at $\approx 80^{\circ} \mathrm{C}$.

efficient charge transport across the thickness of the film in the direction of the $\pi-\pi$ stacking. ${ }^{39}$ On silicon, the first (100) diffraction occurred at $\approx 195{ }^{\circ} \mathrm{C}( \pm 5)$ along the $z$ axis (Table 1 ), consistent with edge-on lamellae formation. However, on graphene the first diffraction peak appeared from (100) along the $x y$ axis (Table 1), indicating the formation of face-on lamellae. Moreover, the face-on crystallites formed at a higher temperature $\left(\approx 200{ }^{\circ} \mathrm{C}( \pm 5)\right)$. With further cooling to $100{ }^{\circ} \mathrm{C}$, the diffraction intensity $I_{100}(x y)$ became much more intense on graphene $\left(\approx 45\right.$ times more at $\left.100{ }^{\circ} \mathrm{C}\right)$, whereas it stayed very weak on silicon, indicating strong vertical $\pi$ stacking on graphene. During cooling, edge-on lamellae grew and became
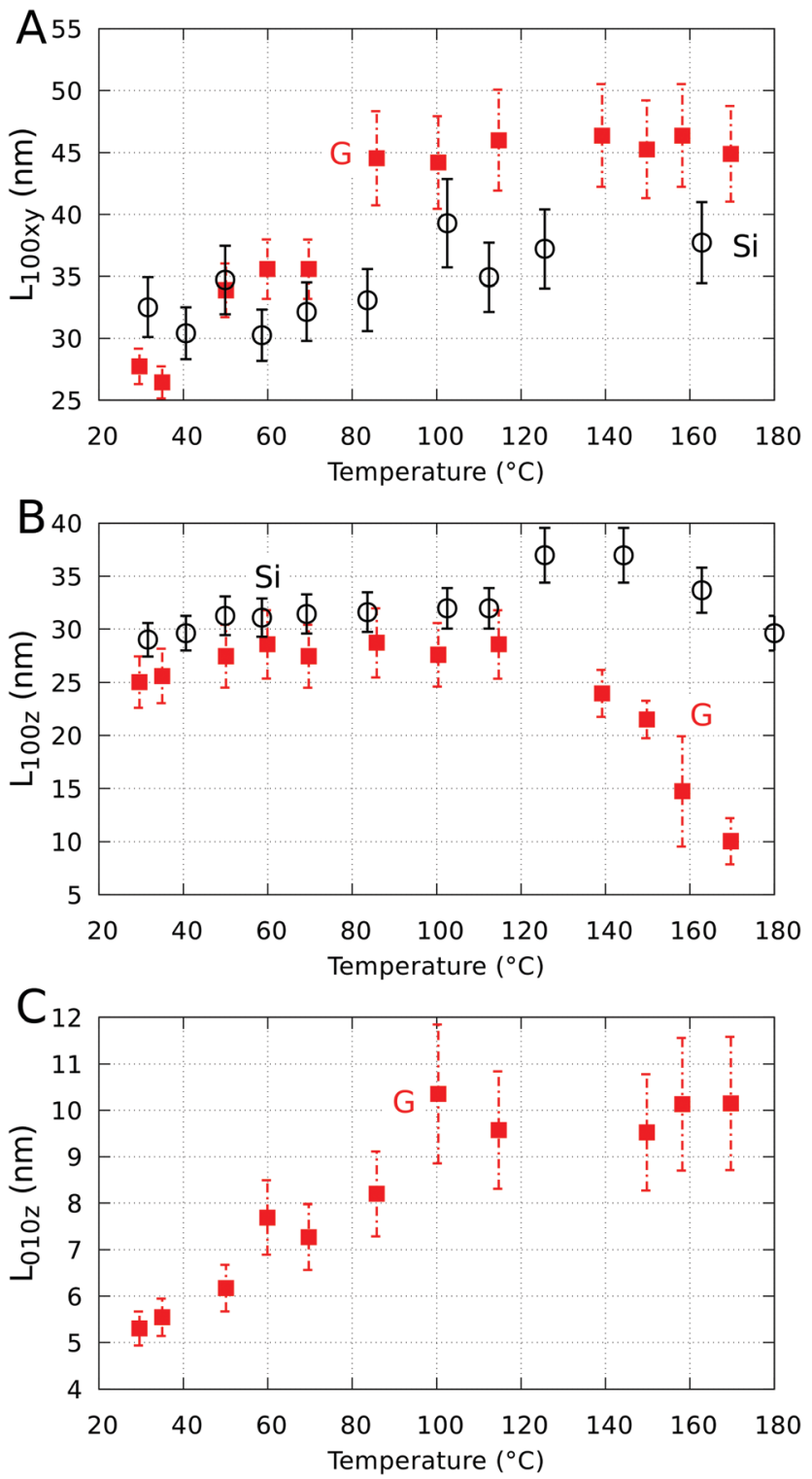

Fig. 6 Evolution of the coherence length during cooling for (A) the $100_{x y}$, (B) the $100_{z}$ and (C) $010_{z}$ on both silicon (black circles) and graphene (red squares). The coherence length decreased with temperature for the $100_{x y}$ peak on both graphene and $\mathrm{Si}$ substrate and for the $01 \mathrm{O}_{z}$ peak on graphene, while $L_{100_{z}}$ increased until it stabilizes at $\approx 120{ }^{\circ} \mathrm{C}$ before decreasing slightly when the temperature reached $\approx 60{ }^{\circ} \mathrm{C}$.

more numerous on both samples, but $I_{100}(z)$ was much more intense on silicon at all temperatures, reaching $\approx 10$ times more intensity at $100{ }^{\circ} \mathrm{C}$ compared to its value at $195{ }^{\circ} \mathrm{C}$. Moreover, the overall degree of crystallinity of the P3HT film, as the integrated intensity of the 100 peak on the whole $\chi$ range (from the $x y$ plane to the $z$ axis), was much lower on graphene $\left(\approx 40\right.$ a.u. at $30{ }^{\circ} \mathrm{C}$ ) compared to the one on silicon (100 a.u. at $30{ }^{\circ} \mathrm{C}$ ). It should also be noted that film thickness is also an important parameter which will likely change the crystallization dynamics, and therefore the results obtained here may not be representative of films which are much thicker or much thinner than the ones used here. 
Table 1 Interplanar distance $d$ and coherence length $L$ for the P3HT films on graphene and silicon at the onset temperature and at room temperature. The crystallization occurs on the graphene substrate at higher temperatures than for the silicon substrate

\begin{tabular}{|c|c|c|c|c|c|}
\hline & Onset temperature $\left({ }^{\circ} \mathrm{C}\right)$ & \multicolumn{2}{|l|}{ At the onset } & \multicolumn{2}{|l|}{ At $30^{\circ} \mathrm{C}$} \\
\hline $\mathrm{G} 100_{z}$ & $185( \pm 5)$ & $1.97( \pm 0.03)$ & $7.41( \pm 1.12)$ & $1.74( \pm 0.02)$ & $25.03( \pm 2.43)$ \\
\hline G $010_{z}$ & $195( \pm 5)$ & $0.383( \pm 0.001)$ & $9.81( \pm 3.42)$ & $0.374( \pm 0.001)$ & $5.29( \pm 0.86)$ \\
\hline Si $100_{z}$ & $195( \pm 5)$ & $2.01( \pm 0.03)$ & $6.87( \pm 1.64)$ & $1.75( \pm 0.02)$ & $29.01( \pm 1.57)$ \\
\hline Si $100_{x y}$ & $163-180^{a}$ & $1.75( \pm 0.02)$ & $44.9( \pm 7.18)$ & $1.65( \pm 0.01)$ & $32.51( \pm 5.20)$ \\
\hline
\end{tabular}

${ }^{a}$ The values given for $d$ and $L$ at the onset temperature are fitted from the data at $163{ }^{\circ} \mathrm{C}$.

\section{Conclusions}

In conclusion, we have performed an in situ study of the crystalline structure of P3HT films on both silicon and graphene substrates as a function of the cooling temperature from the melt. Our results show that $\pi-\pi$ interactions between P3HT and graphene promote coplanar molecular arrangement of the $\mathrm{P} 3 \mathrm{HT}$ chains on the graphene surface, which favor the nucleation of face-on lamellae at a relatively high temperature between 185-200 ${ }^{\circ} \mathrm{C}$. On silicon however, crystallization of edge-on lamellae was strongly favored due to the weaker interactions with the substrate. The overall degree of crystallinity of the film on graphene was only $40 \%$ of that of the film on silicon, and the final ratio of face-on to edge-on lamellae was only $2 \%$ on silicon, and much higher on graphene $(\approx 40 \%)$. Large differences in the crystallite coherence length were also measured on both surfaces, with a surprising reduction in face-on coherence length by $50 \%$ on graphene during cooling. These results provide a detailed study of how semi-conducting polymer films crystallize on graphene, and they may help better control their optoelectronic properties by a better understanding of the crystallization process and kinetics on both a weakly and a more strongly interacting surface.

\section{Acknowledgements}

Portions of this research were carried out at the Stanford Synchrotron Radiation Lightsource, a Directorate of SLAC National Accelerator Laboratory and an Office of Science User Facility operated for the U.S. Department of Energy Office of Science by Stanford University. Some sample preparation was performed at MyFab (Ångström Laboratory) at Uppsala University. The authors thank the Baltic Foundation and the Kempe Foundation for financial support. Special thanks go to Vasyl Skrypnychuk for help with the sample preparation, and discussions.

\section{References}

1 J. D. Buron, D. H. Petersen, P. Bøggild, D. G. Cooke, M. Hilke, J. Sun, E. Whiteway, P. F. Nielsen, O. Hansen, A. Yurgens and P. U. Jepsen, Nano Lett., 2012, 12, 5074-5081.

2 I. P. Murray, S. J. Lou, L. J. Cote, S. Loser, C. J. Kadleck, T. Xu, J. M. Szarko, B. S. Rolczynski, J. E. Johns, J. Huang,
L. Yu, L. X. Chen, T. J. Marks and M. C. Hersam, J. Phys. Chem. Lett., 2011, 2, 3006-3012.

3 X.-C. Jiang, Y.-Q. Li, Y.-H. Deng, Q.-Q. Zhuo, S.-T. Lee and J.-X. Tang, Appl. Phys. Lett., 2013, 103, 073305.

4 L. Zhang, Y. Li, J. Shi, G. Shi and S. Cao, Mater. Chem. Phys., 2013, 142, 626-632.

5 K. S. Novoselov, A. K. Geim, S. V. Morozov, D. Jiang, Y. Zhang, S. V. Dubonos, I. V. Grigorieva and A. A. Firsov, Science, 2004, 306, 666-669.

6 B. Obradovic, R. Kotlyar, F. Heinz, P. Matagne, T. Rakshit, M. D. Giles, M. A. Stettler and D. E. Nikonov, Appl. Phys. Lett., 2006, 88, 14202.

7 Z. Liu, Q. Liu, Y. Huang, Y. Ma, S. Yin, X. Zhang, W. Sun and Y. Chen, Adv. Mater., 2008, 20, 3924-3930.

8 Y.-J. Jeon, J.-M. Yun, D.-Y. Kim, S.-I. Na and S.-S. Kim, Sol. Energy Mater. Sol. Cells, 2012, 105, 96-102.

9 V. Skrypnychuk, N. Boulanger, V. Yu, M. Hilke, S. C. B. Mannsfeld, M. F. Toney and D. R. Barbero, Adv. Funct. Mater., 2015, 25, 664-670.

10 D. H. Kim, H. S. Lee, H.-J. Shin, Y.-S. Bae, K.-H. Lee, S.-W. Kim, D. Choi and J.-Y. Choi, Soft Matter, 2013, 9, 5355-5360.

11 Y. Tong, Y. Lin, S. Wang and M. Song, Polymer, 2015, 73, 52-61.

12 Y. Wang, J. A. Torres, A. Z. Stieg, S. Jiang, M. T. Yeung, Y. Rubin, S. Chaudhuri, X. Duan and R. B. Kaner, ACS Nano, 2015, 9, 9486-9496.

13 J. Y. Choi, W. Kang, B. Kang, W. Cha, S. K. Son, Y. Yoon, H. Kim, Y. Kang, M. J. Ko, H. J. Son, K. Cho, J. H. Cho and B. Kim, ACS Appl. Mater. Interfaces, 2015, 7, 6002-6012.

14 R. Joseph Kline, M. D. McGehee and M. F. Toney, Nat. Mater., 2006, 5, 222-228.

15 N. Kayunkid, S. Uttiya and M. Brinkmann, Macromolecules, 2010, 43, 4961-4967.

16 E. Verploegen, R. Mondal, C. J. Bettinger, S. Sok, M. F. Toney and Z. Bao, Adv. Funct. Mater., 2010, 20, 3519-3529.

17 D. R. Cooper, B. D’Anjou, N. Ghattamaneni, B. Harack, M. Hilke, A. Horth, N. Majlis, M. Massicotte, L. Vandsburger, E. Whiteway and V. Yu, ISRN Condens. Matter Phys., 2012, 501686.

18 G. Wang, J. Swensen, D. Moses and A. J. Heeger, J. Appl. Phys., 2003, 93, 6137-6141.

19 H. Yang, T. Shin, L. Yang, K. Cho, C. Ryu and Z. Bao, Adv. Funct. Mater., 2005, 15, 671-676.

20 M. Surin, P. Leclère, R. Lazzaroni, J. D. Yuen, G. Wang, D. Moses, A. J. Heeger, S. Cho and K. Lee, J. Appl. Phys., 2006, 100, 033712 . 
21 D. Gargi, R. J. Kline, D. M. DeLongchamp, D. A. Fischer, M. F. Toney and B. T. O'Connor, J. Phys. Chem. C, 2013, 117, 17421-17428.

22 L. H. Jimison, S. Himmelberger, D. T. Duong, J. Rivnay, M. F. Toney and A. Salleo, J. Polym. Sci., Part B: Polym. Phys., 2013, 51, 611-620.

23 T. Salammal Shabi, S. Grigorian, M. Brinkmann, U. Pietsch, N. Koenen, N. Kayunkid and U. Scherf, J. Appl. Polym. Sci., 2012, 125, 2335-2341.

24 G. Scavia, W. Porzio, S. Destri, L. Barba, G. Arrighetti, S. Milita, L. Fumagalli, D. Natali and M. Sampietro, Surf. Sci., 2008, 602, 3106-3115.

25 D. M. DeLongchamp, R. J. Kline, Y. Jung, E. K. Lin, D. A. Fischer, D. J. Gundlach, S. K. Cotts, A. J. Moad, L. J. Richter, M. F. Toney, M. Heeney and I. McCulloch, Macromolecules, 2008, 41, 5709-5715.

26 S. Obata and Y. Shimoi, Phys. Chem. Chem. Phys., 2013, 15, 9265.

27 Z. Bartczak, A. Argon, R. Cohen and T. Kowalewski, Polymer, 1999, 40, 2367-2380.

28 S. Lilliu, T. Agostinelli, E. Pires, M. Hampton, J. Nelson and J. E. Macdonald, Macromolecules, 2011, 44, 2725-2734.

29 C. R. Singh, G. Gupta, R. Lohwasser, S. Engmann, J. Balko, M. Thelakkat, T. Thurn-Albrecht and H. Hoppe, J. Polym. Sci., Part B: Polym. Phys., 2013, 51, 943-951.
30 J. Yang, Y. Li, S. Duhm, J. Tang, S. Kera and N. Ueno, Adv. Mater. Interfaces, 2014, 1, 1300128.

31 K. A. Smith, Y.-H. Lin, J. W. Mok, K. G. Yager, J. Strzalka, W. Nie, A. D. Mohite and R. Verduzco, Macromolecules, 2015, 48, 8346-8353.

32 S. Fall, L. Biniek, Y. Odarchenko, D. V. Anokhin, G. de Tournadre, P. Leveque, N. Leclerc, D. A. Ivanov, O. Simonetti, L. Giraudet and T. Heiser, J. Mater. Chem. C, 2016, 4, 286-294.

33 K. Kanai, T. Miyazaki, H. Suzuki, M. Inaba, Y. Ouchi and K. Seki, Phys. Chem. Chem. Phys., 2010, 12, 273-282.

34 G. L. Destri, T. F. Keller, M. Catellani, F. Punzo, K. D. Jandt and G. Marletta, Macromol. Chem. Phys., 2011, 212, 905-914.

35 M. F. Toney and S. Brennan, Phys. Rev. B: Condens. Matter Mater. Phys., 1989, 39, 7963-7966.

36 X. Shen, V. V. Duzhko and T. P. Russell, Adv. Energy Mater., 2013, 3, 263-270.

37 K. A. Mazzio, A. H. Rice, M. M. Durban and C. K. Luscombe, J. Phys. Chem. C, 2015, 119, 14911-14918.

38 H. G. O. Sandberg, G. L. Frey, M. N. Shkunov, H. Sirringhaus, R. H. Friend, M. M. Nielsen and C. Kumpf, Langmuir, 2002, 18, 10176-10182.

39 V. Skrypnychuk, N. Boulanger, V. Yu, M. Hilke, M. F. Toney and D. R. Barbero, J. Mater. Chem. C, 2016, 4, 4143-4149. 